\title{
Adaptive Noise Cancellation for speech Employing Fuzzy and Neural Network
}

\author{
Mohammed Hussein Miry \\ Dep. of Electrical and Electronic Eng. \\ University of Technology \\ Baghdad/Iraq \\ Email:Mohammed_miry@yahoo.Com
}

\author{
Ali Hussien Mary \\ Al-Khwarizmy College. Of Eng. \\ University of Baghdad \\ Baghdad /Iraq \\ Email: Alihussien76@yahoo.Com
}

\author{
Hussain Kareem Khleaf \\ Dep.of Electrical and Electronic Eng. \\ University of Technology \\ Baghdad /Iraq \\ Email: eng_h_k_msc@yahoo.Com
}

\begin{abstract}
Adaptive filtering constitutes one of the core technologies in digital signal processing and finds numerous application areas in science as well as in industry. Adaptive filtering techniques are used in a wide range of applications such as noise cancellation. Noise cancellation is a common occurrence in today telecommunication systems. The LMS algorithm which is one of the most efficient criteria for determining the values of the adaptive noise cancellation coefficients are very important in communication systems, but the LMS adaptive noise cancellation suffers response degrades and slow convergence rate under low Signal-toNoise ratio (SNR) condition. This paper presents an adaptive noise canceller algorithm based fuzzy and neural network. The major advantage of the proposed system is its ease of implementation and fast convergence. The proposed algorithm is applied to noise canceling problem of long distance communication channel. The simulation results showed that the proposed model is effectiveness.
\end{abstract}

\section{INTRODUCTION}

During the last years, the fuzzy neural network approach has gained considerable interest for solving real world problems, including modeling and control of highly complex systems, signal processing and pattern recognition [1-4]. Extensive experimentation has demonstrated that the class of feed-forward fuzzy neural networks exhibits a number of significant advantages compared to the neural network models [3,4]. First, the neural networks are global models where training is performed on the entire pattern range. On the contrary, owing to the partition of the input space, the fuzzy models perform a fuzzy blending of local models in space. As a result, faster convergence is achieved during learning for a specific task. Secondly, fuzzy neural networks are capable of incorporating both numerical data (quantitative information) and expert's knowledge (qualitative information) and describe them in the form of linguistic IF THEN rules. In that respect, they provide a unified framework for integrating the computational parallelism and low-level learning of neural networks with the high-level reasoning of fuzzy systems. The above feature assists to determining the initial structure, also leading to models with fewer parameters compared to neural networks. The goal of speech denoising is to improve the quality of a noisy speech signal. In the mobile environment in case of using hands-free accessories, the microphone is situated as far as $1 \mathrm{~m}$ away from the speaker, so that signal processing methods to suppress the background noise and the acoustic echo cancellation for the far-end speaker become necessary [5]. For noise reduction, adaptive noise cancellation is considered with the enhancement of noise- corrupted signal. According to the reference input, an adaptive filter produces a replica of noise and subtracts it from the primary input.

\section{THE ADAPTIVE NOISE CANCELLATION SYSTEM}

In general, the noisy speech signal is given by $\mathrm{x}(\mathrm{k})=\mathrm{s}(\mathrm{k})+\mathrm{n}(\mathrm{k})$, where $\mathrm{s}(\mathrm{k})$ and $\mathrm{n}(\mathrm{k})$ represent the clean speech and noise signal, respectively. Adaptive Noise Cancellation is used to remove noise from useful signals. This is a useful technique where a signal is submerged in a noisy environment [6]. The process of using the adaptive filter for solving the adaptive noise cancellation problem is given in Fig. 1. The information signal $\mathrm{x}$ was recorded with corrupting noise $\mathrm{n}$ that was generated from another noise source through an unknown nonlinear process. The noise $\mathrm{n}$ is Gaussian with zero mean and unit variance. The purpose of adaptive noise cancellation is to produce an anti-wave whose magnitude is exactly the same as that of the unwanted noise and whose phase is exactly opposite. The scheme of adaptive noise cancellation system is shown as Fig 1. The primary input source receives the desired signal $\mathrm{s}(\mathrm{k})$ with corrupting noise $\mathrm{y}(\mathrm{k})$. The corrupting noise $y(k)$ is generated by the noise source $n(k)$. The 
received signal of primary input is thus $\mathrm{x}(\mathrm{k})=\mathrm{s}(\mathrm{k})+\mathrm{y}(\mathrm{k})$. A secondary (reference) input source receives a noise $\mathrm{n}(\mathrm{k})$ uncorrelated with the signal source $\mathrm{s}(\mathrm{k})$ but correlated with the corrupting noise $y(k)$. This secondary input source provides the reference input to the adaptive noise canceller. The $\mathrm{n}(\mathrm{k})$ is used by an adaptive process to generate an output $\hat{y}(k)$ that a replica of $\mathrm{y}(\mathrm{k})$. The output is then subtracted from the primary input $\mathrm{x}(\mathrm{k})$ to recover the desired signal $\mathrm{s}(\mathrm{k})$. The basic assumptions for the adaptive noise cancellation system include:

1. The $\mathrm{s}(\mathrm{k}), \mathrm{n}(\mathrm{k})$ and $\mathrm{y}(\mathrm{k})$ are stationary zero mean processes.

2. The $\mathrm{s}(\mathrm{k})$ is uncorrelated with $\mathrm{n}(\mathrm{k})$ and $\mathrm{y}(\mathrm{k})$.

3. The $\mathrm{n}(\mathrm{k})$ is correlated with $\mathrm{y}(\mathrm{k})$.

4. The $\hat{y}(k)$ are uncorrelated with $\mathrm{s}(\mathrm{k})$.

From Fig. 1, it follows

$$
\hat{s}(k)=s(k)+n(k)-\hat{y}(k)
$$

then the remaining error $\hat{s}(k)$ is exactly the same as the desired signal $\mathrm{s}(\mathrm{k})$.

\section{A. The LMS Adaptation Algorithm}

The least mean squares (LMS) algorithm is used in an adaptive noise cancellation scheme. Given the input vector $\mathrm{x}(\mathrm{k})$, for adjusting the adaptive noise cancellation coefficients at time instant $\mathrm{n}$ is defined as follows:

$$
W(k+1)=W(k)+2 \mu e(k) x(k)
$$

where $\mathrm{k}$ is the iteration index, the $\mathrm{e}(\mathrm{k})$ is defined as $\mathrm{e}(\mathrm{k})=\mathrm{d}(\mathrm{k})+\mathrm{n}(\mathrm{k})-\mathrm{x}^{\mathrm{T}}(\mathrm{k}) \mathrm{W}(\mathrm{k}), \mathrm{d}(\mathrm{k})$ is the desired value and $\mathrm{n}(\mathrm{k})$ is the additive noise. Because of its simplicity, the Least Mean Square (LMS) algorithm

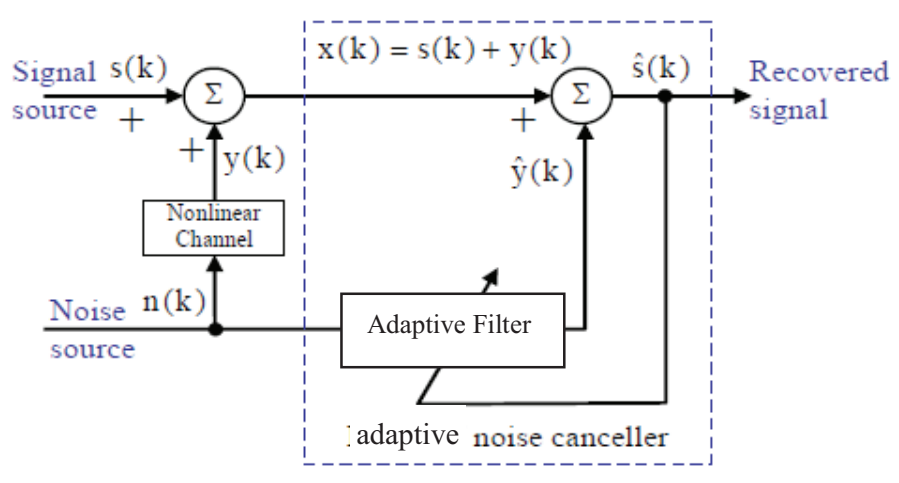

Figure 1: adaptive noise cancellation system is the most popular adaptive algorithm. However, the LMS algorithm suffers from slow and datadependent convergence behavior.

\section{ADAPTIVE NEURO-FUZZY INTERENCE SYSTEM (ANFIS)}

The Adaptive Neuro-Fuzzy Inference System, first introduced by Jang, is a universal approximator and as such is able to approximate any real continuous function on a compact set to any degree of accuracy. Thus, in estimating parameters where the given data are such that the system associates measurable system variables with an internal system parameter, functional mapping can be constructed by ANFIS to approximate the process of estimation of the internal system parameter. ANFIS is a neuro-fuzzy system that combines the learning capabilities of neural networks, with the functionality of fuzzy inference systems [6].The ANFIS is functionally equivalent to fuzzy inference systems. The hybrid learning algorithm which combines gradient descent and the least-squares method are briefly discussed below .

\section{A. Artificial Neural Networks (ANN)}

ANNs are mathematical modeling tools which are particularly useful for predicting and forecasting in complex settings. Historically, these were meant to simulate, at a simplified level, the activity of the human brain. The ANN consists of an input layer, an output layer, and one or more hidden layers as shown in Fig. 2, the ANN accomplishes this through a large number of highly interconnected processing elements (neurons), working in unison to solve specific problems, such as forecasting and pattern recognition. Each neuron is connected to some of its neighbors with varying coefficients or

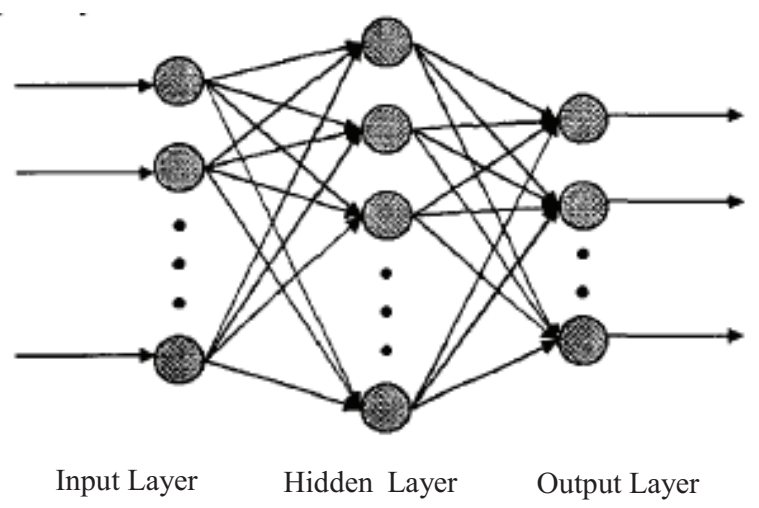

Figure 2: The Neural Network 
weights which represent the relative influence of the different neuron inputs on other neurons.

\section{B. Fuzzy Systems}

Fuzzy systems are made of a knowledge base and reasoning mechanism called fuzzy inference system. A fuzzy inference system combines fuzzy ifthen rules into a mapping from the inputs of the system into its outputs, using fuzzy reasoning methods. That is, fuzzy systems represents nonlinear mapping accompanied by fuzzy if-then rules from the rule base. Each of these rules describes the local mappings. The rule base can be constructed either from human expert or automatic generation that is extraction of rules using numerical input-output data [7]. A fuzzy inference system (FIS) consists of four functional as shown in Fig. 3:

- Fuzzification: transforms the crisp inputs into degrees of match with linguistic values.

- Knowledge base: consists of a rule base and a database. A rule base contains a number of fuzzy if-then rules. A database defines the membership function of the fuzzy sets used in the fuzzy rules.

- Fuzzy inference engine: performs the inference operations on the rules.

- Defuzzification: transforms the fuzzy results of the inference into a crisp output.

As a simple example, a fuzzy inference system with two inputs $\mathrm{x}$ and $\mathrm{y}$ and one output $\mathrm{z}$ is assumed. The first-order Sugeno fuzzy model, a typical rule set with two fuzzy If-Then rules, can be expressed as: Rule 1 : IF $\mathrm{x}$ is $\mathrm{A}_{1}$ AND $\mathrm{y}$ is $\mathrm{B}_{1}$ THEN

$$
f_{1}=p_{1} x+q_{1} y+r_{1}
$$

Rule 2 : IF $\mathrm{x}$ is $\mathrm{A}_{2}$ AND $\mathrm{y}$ is $\mathrm{B}_{2}$ THEN

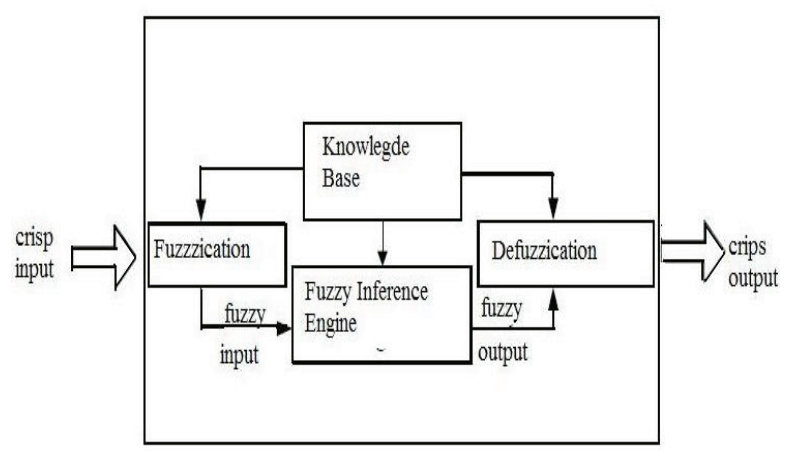

Figure 3: Fuzzy Inference Engine

$$
f_{2}=p_{2} x+q_{2} y+r_{2}
$$

The resulting Sugeno fuzzy reasoning system is shown in Fig. 4. Here, the output $\mathrm{z}$ is the weighted average of the individual rule outputs and is itself a crisp value.

\section{ANFIS Architecture}

ANFIS structure is a generalized neural network using a Sugeno fuzzy model, as illustrated in Fig. 5 [8-10]. Nodes at the same layer have similar functions. The output of the ith node in layer $l$ is denoted $O_{l, i}$

Layer 1: Every node $\mathrm{i}$ in this layer is an adaptive node with a node function

$$
\begin{array}{ll}
O_{l, i}=\mu A_{i}(x) & \text { for } \mathrm{i}=1,2 \text { or } \\
O_{l, i}=\mu B_{i-2}(y) & \text { for } \mathrm{i}=3,4
\end{array}
$$

where $\mathrm{x}$ (or $\mathrm{y}$ ) is the input to the ith node and $\mathrm{A}_{\mathrm{i}}$ (or $\mathrm{B}_{\mathrm{i}-2}$ ) is a linguistic label (such as "low" or "high") associated with this node. In other words, $O_{l, i}$ is the membership grade of a fuzzy set $\mathrm{A}\left(=\mathrm{A}_{1}, \mathrm{~A}_{2}, \mathrm{~B}_{1}\right.$, or $B_{2}$ ) and it specifies the degree to which the given input $\mathrm{x}$ (or $\mathrm{y}$ ) satisfies the quantifier $\mathrm{A}$. The membership functions for A and B are usually described by generalized bell functions, e.g.:

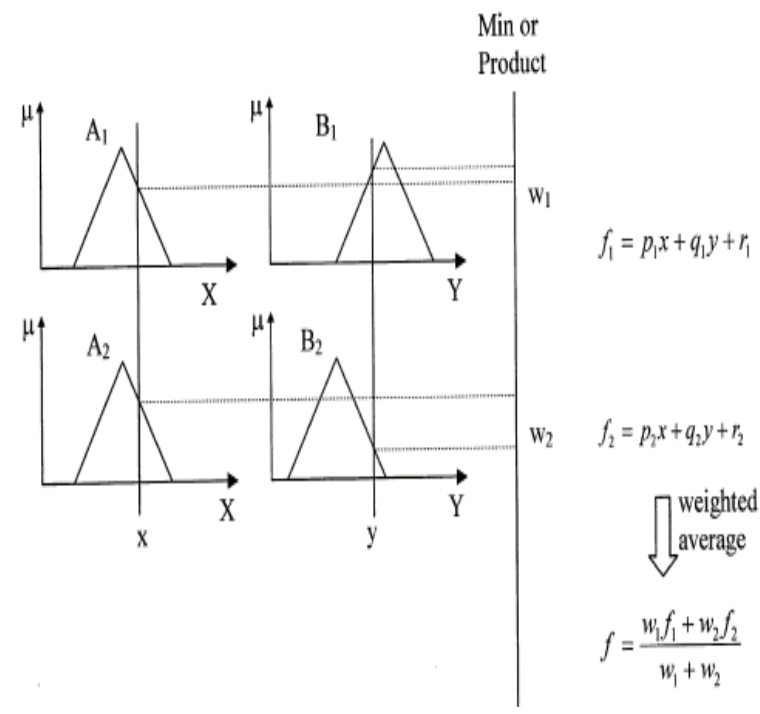

Figure 4: Two-input first-order Sugeno fuzzy model with two rules 


$$
\mu A_{i}(x)=\frac{1}{1+\left[\left(x-c_{i}\right) / a_{i}\right]^{2 b_{i}}}
$$

where $\left\{a_{i}, b_{i}, c_{i}\right\}$ is the parameter set. As the values of these parameters change, the bell-shaped function varies accordingly, exhibiting various forms of membership functions on the linguistic label $\mathrm{A}_{\mathrm{i}}$. In fact, any continuous and piecewise differentiable functions, such as the commonly used triangularshaped membership functions, are also qualified candidates for node functions in this layer. Parameters in this layer are referred to as premise parameters. The outputs of this layer are the membership values of the premise part.

Layer 2: This layer consists of the nodes labeled $\Pi$ which multiply incoming signals and send the product out. For instance,

$$
O_{l, i}=w_{i}=\mu A_{i}(x) \mu B_{i}(y) \quad \mathrm{i}=1,2
$$

Each node output represents the firing strength of a rule.

Layer 3: In this layer, the nodes labeled $\mathrm{N}$ calculate the ratio of the ith rule's firing strength to the sum of all rules' firing strengths

$$
O_{3, i}=\overline{w_{i}}=\frac{w_{i}}{w_{1}+w_{2}} \quad \mathrm{i}=1,2
$$

The outputs of this layer are called the normalized firing strengths.

Layer 4: This layer's nodes are adaptive with node Functions

$$
O_{4, i}=\overline{w_{i}} f_{i}=\overline{w_{i}}\left(p_{i} x+q_{i} y+r_{i}\right)
$$

where $\overline{w_{i}}$ is the output of layer 3 , and $\left\{\mathrm{p}_{\mathrm{i}}, \mathrm{q}_{\mathrm{i}}, \mathrm{r}_{\mathrm{i}}\right\}$ are the parameter set. Parameters of this layer are referred to as consequent parameters.

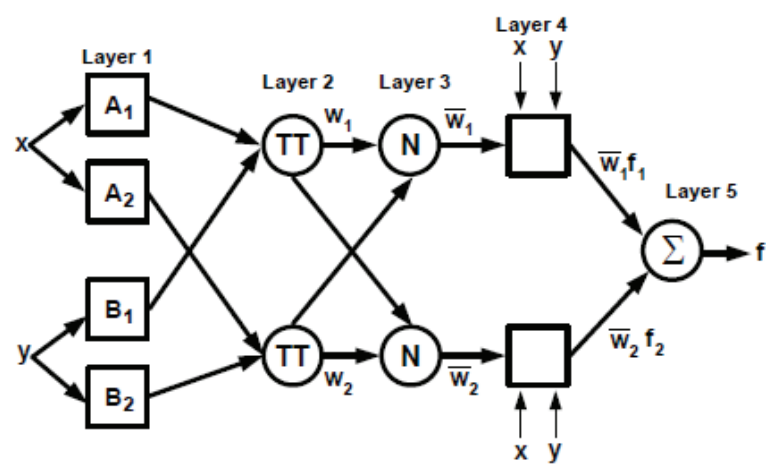

Figure 5: Equivalent ANFIS Architecture
Layer 5: This layer's single fixed node, labeled $\Sigma$, computes the final output as the summation of all the incoming signals

$$
O_{5, i}=\sum_{i=1} \overline{w_{i}} f_{i}=\frac{\sum_{i} w_{i} f_{i}}{\sum_{i} w_{i}}
$$

Thus, an adaptive network that is functionally equivalent toa Sugeno first-order fuzzy inference system is created. The flowchart of ANFIS is shown in Fig. 6.

\section{PROPOSED MODEL}

The proposed system for speech denoising based adaptive nose cancellation and ANFIS shown in Fig. 7. The main procedure of the system is described as follows:

1. Generate white Gaussian noise $n(k)$. ( $-4 \mathrm{~dB}, 0 \mathrm{~dB}$, $4 \mathrm{~dB})$

2. Generate noise $y(k)$ from $n(k)$ by nonlinear channel.

3. The measured signal $x(k)$ is the sum of speech original signal $s(k)$ and noise $y(k)$.

4. Noise $n(k)$ passes through the ANFIS adaptive filter to produce an output $\hat{y}(k)$

5. The ANFIS estimated $\hat{y}(k)$ after training, $\hat{y}(k)$ is replica of $y(k)$

6. the proposed model estimated desired speech signal by equation

$$
\hat{s}(k)=s(k)+y(k)-\hat{y}(k)
$$

\section{SIMULATION AND RESULT}

Base on the purposed technique, the measurable output noisy speech signal with SNR of different level (low noise, medium noise, and high noise ) are taken as the contaminated version level of the interference, since the noise is not directly available. The white noise source is taken as the input of adaptive filter. The ANFIS output $\hat{y^{\wedge}(k)}$ produced from the adaptive filter is a close replica of $y(k)$. In purposed technique, Parameter settings for the ANFIS algorithm are the training epoch number is 10 , the number of MFs for each input is 4 , the initial step size, step size decrease rate, and step size increase rate are $0.1,0.9$, and 1.1 , respectively, the learning algorithm is hybrid learning algorithm and different types of membership function are used to 


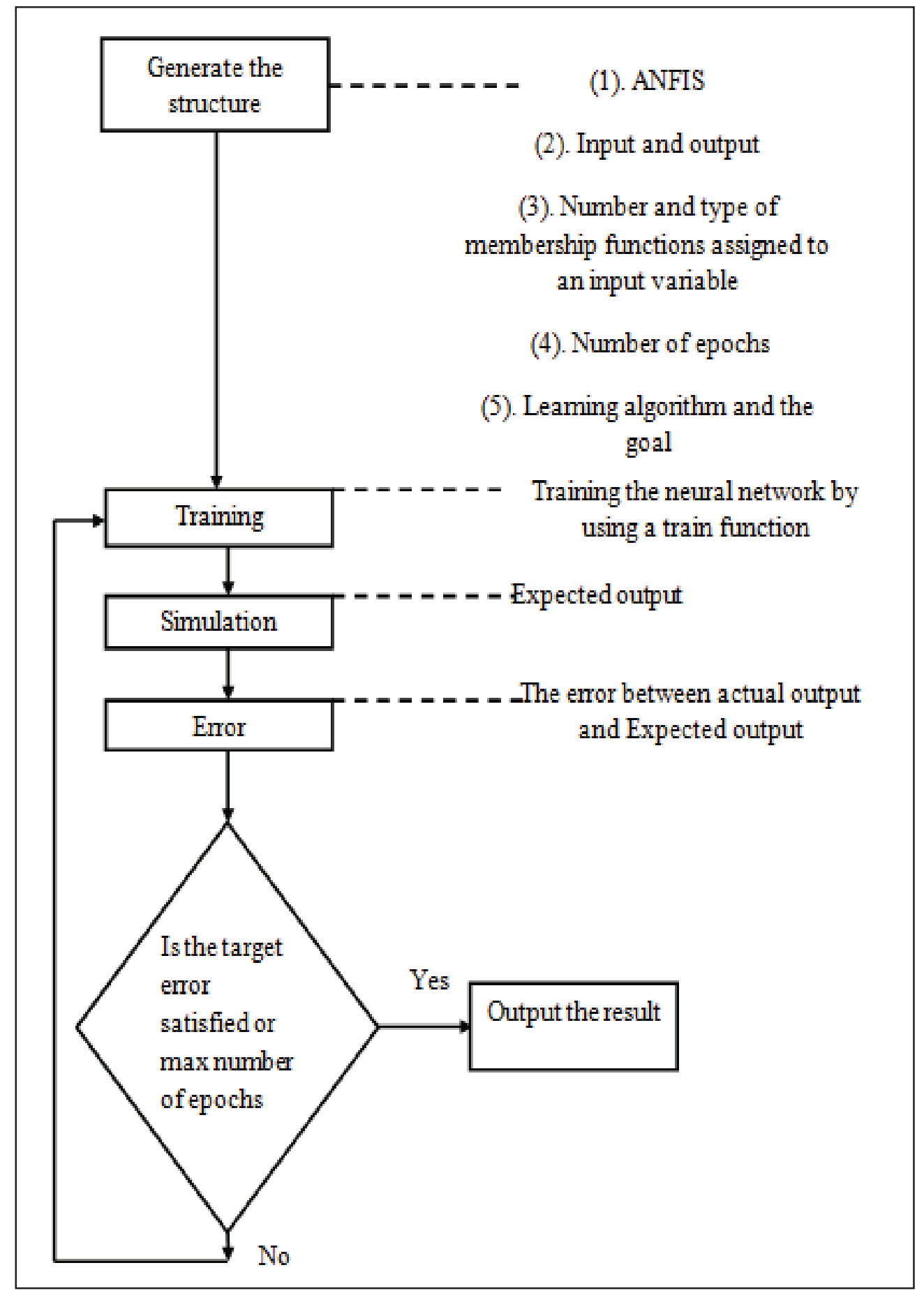

Figure 6: Flowchart of ANFIS 
improve SNR for noise speech. The relation of signal to noise ratio, defined it as shown in "(10)", Table I shows the SNR enhancement results by using purposed model

$$
S N R_{\text {out }}=10 \log \frac{\sum_{n=1}^{K} s^{2}(n)}{\sum_{n=1}^{k}[s(n)-\hat{s}(n)]^{2}}
$$

From this table, we notice the proposed model with membership function of Triangular type given higher SNR than other types of membership function. The simulation results are illustrated in Fig. 8, Fig. 9 and Fig. 10. As illustrated in Fig.8, Obviously the effectiveness of ANFIS technique for adaptive noise cancellation was confirmed. Near original speech signal was obtained after noise cancellation. As illustrated in Fig.9 and Fig.10, near original speech signal was also obtained. Performance comparison of ANFIS and LMS in quantitative terms is made in Table II. From the numerical values presented in Table II, it is apparent that the performance of ANFIS, as measured by $\mathrm{SNR}_{\text {out }}$, is significantly better than that of LMS. Fig. 11 shown the comparison between the performance of the proposed method and adaptive noise cancellation by using LMS. Those plots show that convergence of the proposed method is faster than that of LMS. From these simulation results, each recovered Speech signal are agree with each original speech signal. ANFIS technique has enough capability to use as adaptive noise filter for speech noisy signal.

\section{CONCLUSION}

An adaptive noise cancellation technique was studied and used to de-noise speech signal. This paper has compared, through analysis and simulation, proposed system and LMS system for adaptive noise cancellation. In the paper, a new system is proposed for adaptive noise cancellation based Fuzzy and neural network. A new algorithm has been proposed which moderately has two advantages over the LMS algorithm in low SNR environment. One, it is more efficient to eliminate noise. The second, it offers faster convergence time.

\section{REFERENCE}

[1] M.F. Azeem, M. Hanmandlu, "Structure identification of generalized adaptive neuro-fuzzy inference systems', IEEE Trans. Fuzzy Systems 11 (5) (2003) 666-681.

[2] M.F. Azeem, M. Hanmandlu, N. Ahmad, "'Generalization of adaptive neuro-fuzzy inference systems', IEEE Trans. Neural Networks 11 (6) (2000)1332-1346.

[3] S.A. Billings, M. El-Hitmy, "Adaptive noise cancellation for a class of nonlinear IIR filters', J. Sound Vibr. 140 (3) (1990) 457-473.

[4] V. Gorrini, H. Bersini, 'Recurrent fuzzy systems', Proc. IEEE Int. Conf. Fuzzy Systems, vol. 1, 1994, pp. 193-198.

[5] Q. Linmei , H. Guangrui and L. Chongni, “ A New Speech Enhancement Method ", in proc of International symposium on intelligent Multimedia Video and Speech Processing, May 2-4 2001.

[6] B. MARUNGSRI and A. OONSIVILAI ' Fuzzy ARTMAP Technique for Speech Noise Reduction", Proceedings of the 7th WSEAS International Conference on Signal, Speech and Image Processing, Beijing, China, September 15-17, 2007,pp 20-24

[7] Czogala, E., Leski, J.,"Fuzzy and Neuro-Fuzzy Intelligent Systems", Physica- Verlag Heidelberg, New York, 2000

[8] Chia-Feng Juang, Chyi-Tian Chiou, and ChunLung Lai, "'Hierarchical Singleton-Type Recurrent Neural Fuzzy Networks for Noisy Speech Recognition', IEEE Trans. On neural networks, vol. 18, no.. 3, MAY 2007,pp 833-843

[9] J.B. Theocharis, "A high-order recurrent neurofuzzy system with internal dynamics: Application to the adaptive noise cancellation', Fuzzy Sets and Systems 157 (2006) pp.471 - 500

[10] H. Qin and S. X.Yang, "' Adaptive neuro-fuzzy inference systems based approach to nonlinear noise cancellation for images', Fuzzy Sets and Systems 158 (2007) pp.1036 - 1063 


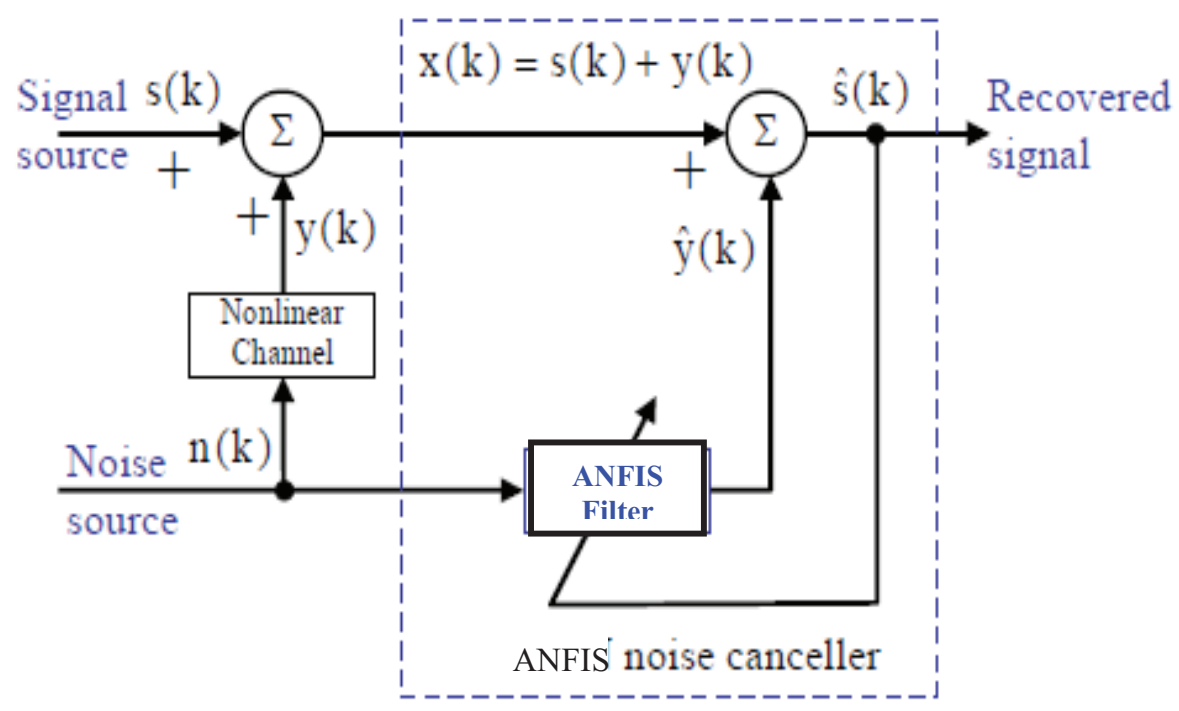

Figure 7: The Proposed Model

Table I

Speech denoising measure obtained by several types of membership function of proposed model for different SNR for speech.

\begin{tabular}{|c|c|c|c|}
\hline \multirow{2}{*}{ Name of MFs } & \multicolumn{3}{|c|}{ SNR(dB) input } \\
\cline { 2 - 4 } & \multicolumn{3}{|c|}{ SNR(dB) output } \\
\cline { 2 - 4 } & \multicolumn{2}{|c|}{} & \multicolumn{2}{c|}{} \\
\hline Triangular & 6.59 & 8.12 & 10.3 \\
\hline Gaussian & 6.32 & 7.86 & 9.47 \\
\hline Trapezoidal & 5.96 & 7.72 & 8.98 \\
\hline Pi-shaped & 3.25 & 5.79 & 7.61 \\
\hline Bell & 5.62 & 7.32 & 7.81 \\
\hline $\begin{array}{c}\text { Difference between two } \\
\text { sigmoidal }\end{array}$ & 4.12 & 6.73 & 7.71 \\
\hline $\begin{array}{c}\text { Two-sided Gaussian } \\
\text { Product of two sigmoid }\end{array}$ & 3.67 & 5.89 & 7.95 \\
\hline
\end{tabular}



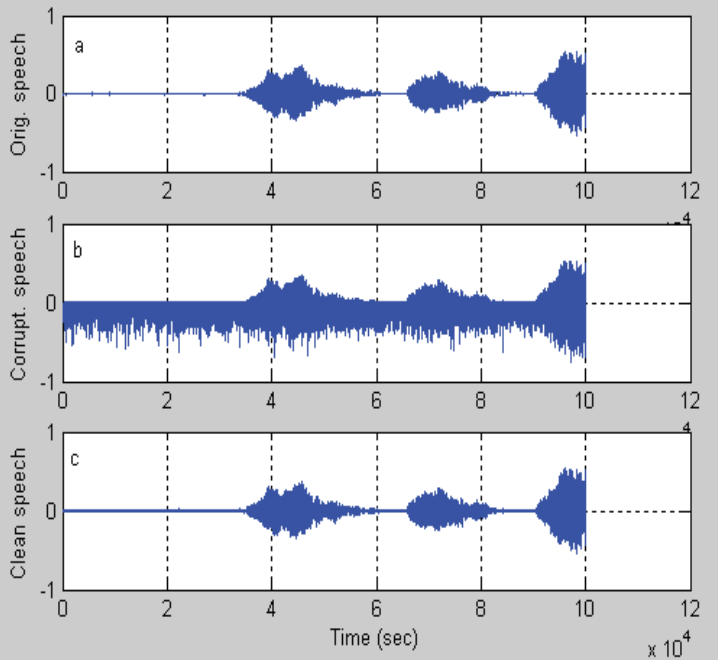

Figure 8: Results of noise cancellation, (a) Clean Speech (b) Noisy Speech (-4 dB), (c) Speech after noise cancellation
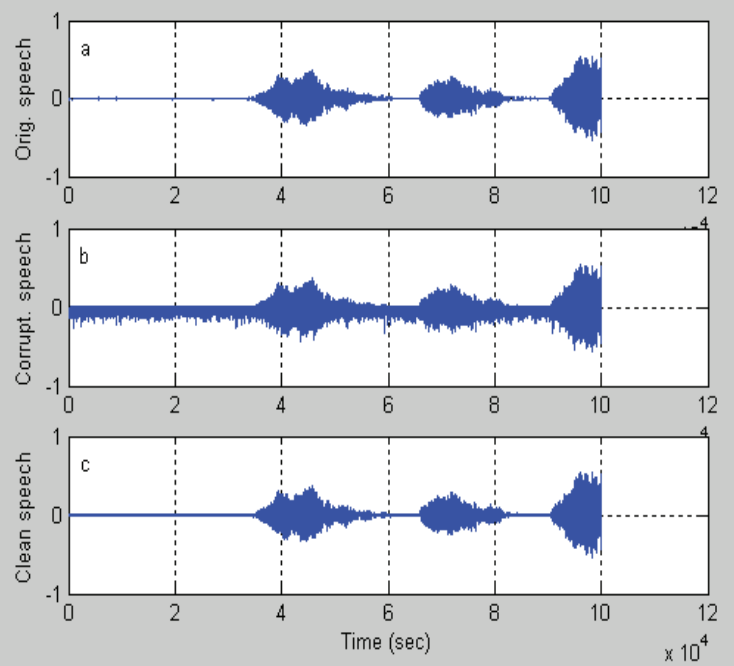

Figure 9: Results of noise cancellation, (a) Clean Speech (b) Noisy Speech (0 dB), (c) Speech after noise cancellation

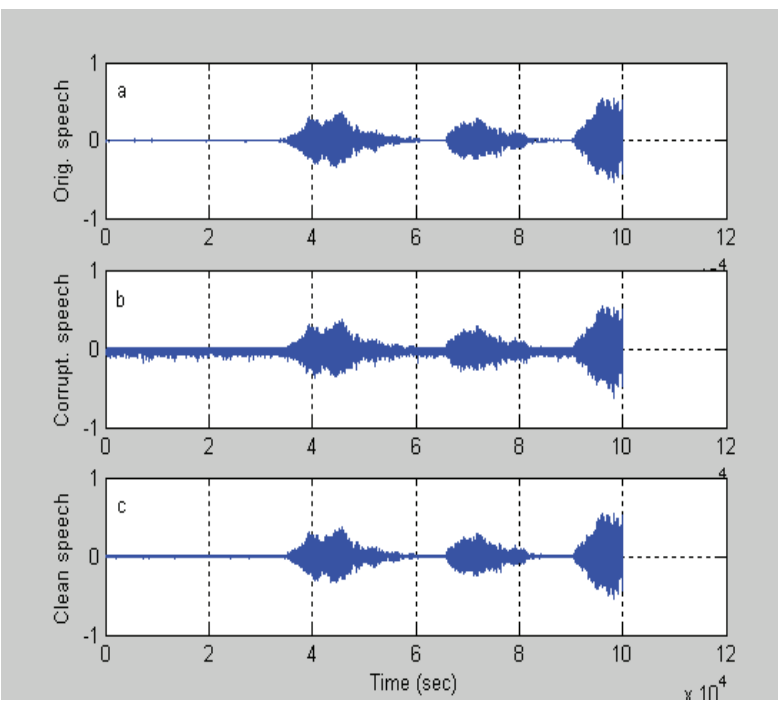

Figure 10: Results of noise cancellation, (a) Clean Speech (b) Noisy Speech (4 dB), (c) Speech after noise cancellation

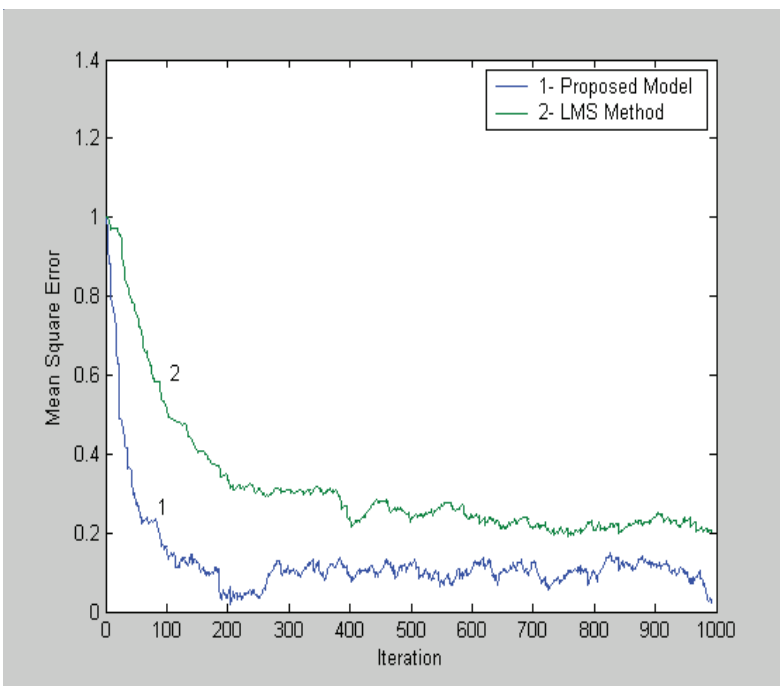

Figure 11: Comparison of Convergence Rate for the LMS System and proposed System with $\mathrm{SNR}_{\text {input }}=4 \mathrm{~dB}$

Table II : Comparison between ANFIS and LMS

\begin{tabular}{|l|c|c|}
\hline & ANFIS & LMS \\
\hline SNR $_{\text {input }}(\mathrm{dB})$ & 4 & 4 \\
\hline SNR $_{\text {output }}(\mathrm{dB})$ & 10.3 & 7.94 \\
\hline
\end{tabular}

